\title{
HACIA UN ENFOQUE SOSTENIBLE DE RETRIBUCIÓN QUE INCLUYA LAS EXPECTATIVAS DE LA POBLACIÓN TRABAJADORA
}

\author{
Autores: Carlos Anta Callersten \\ carlos.anta.callersten@acciona.es \\ Director de Talento y Compensación en ACCIONA \\ María José Martín Rodrigo \\ mariajo@comillas.edu \\ Universidad Pontificia Comillas \\ Antonio Núñez Partido \\ anup@comillas.edu \\ Universidad Pontificia Comillas
}

\section{Resumen}

La guerra por el talento está sofisticando las estrategias de las empresas para atraer y fidelizar a los mejores profesionales. En este contexto, la retribución desempeña un papel destacado. Pero igualmente relevante es su significado cuando se habla de crear y/o aumentar la tasa de empleo decente, como enuncia el objetivo de desarrollo sostenible número 8, definido por Naciones Unidas para el 2030. Por ello, se hace pertinente revisar el concepto de retribución para que contribuya a un crecimiento económico más sostenible e inclusivo. Así, teniendo en cuenta las expectativas que 
la población tiene sobre un trabajo decente, el concepto sobre la retribución se ha ido ampliando desde un enfoque puramente monetario hasta adquirir un significado más amplio que abarca todos los beneficios percibidos por los trabajadores en su relación laboral. En línea con los resultados de algunos estudios realizados al efecto, este trabajo argumenta la conveniencia de incluir dichas expectativas en la definición de las políticas de retribución y, tenerlas en cuenta también, en las decisiones laborales, pues resultan ser altamente motivadoras contribuyendo al aumento de la productividad. Así, tras el análisis de la toma de decisiones en distintas situaciones laborales como la expatriación o, la decisión de cursar un MBA, se argumenta que las expectativas de los trabajadores desempeñan un papel relevante debiendo formar parte de una definición moderna, sostenible e inclusiva de retribución.

Palabras clave: retribución; teoría de las expectativas; contrato psicológico; trabajo decente; objetivo desarrollo sostenible.

\section{Towards a sustainable retribution approach that includes expectations of the working population}

\section{Abstract}

The war for talent is sophisticating companies' strategies to attract and retain the best professionals. In this context, retribution plays an equally important role when it comes to creating and increasing the decent employment rate, as set out in United Nations Sustainable Development Goal 8 by 2030. Therefore, it is pertinent to review the concept of remuneration so that it contributes to more sustainable and inclusive economic growth. Thus, taking into account the expectations that the population has about decent work, the concept of compensation has been extended from a purely monetary approach to acquiring a broader meaning that encompasses all the benefits perceived by workers in their employment relationship. In line with the results of some studies carried out for this purpose, this work argues the desirability of including such expectations in the definition of remuneration policies and, also taking them into account, in labor decisions, since they turn out to be highly motivating contributing to the increase in the productivity. Thus, after the analysis of decision-making in different work situations such as expatriation or the decision to pursue an MBA, it is argued that workers' expectations play an essential role and should be part of a modern, sustainable, and inclusive definition of retribution.

Keywords: compensation, the theory of expectations; psychological contract; decent work; sustainable development goal. 


\section{INTRODUCCIÓN}

La guerra por el talento está sofisticando las estrategias de las empresas para atraer y fidelizar a los mejores profesionales. La retribución desempeña un papel crítico en cualquier estrategia de gestión del talento y, su enfoque, se ha ido ampliando desde un concepto puramente monetario hasta adquirir un significado más amplio que abarca todos los beneficios percibidos por los trabajadores en su relación laboral.

Pero en una etapa de concienciación social, en la que las empresas no miran solo su cuenta de resultados sino también su impacto medioambiental y social, hacia un mundo más sostenible e inclusivo, cuyas metas pretendan lograr una globalización justa, la reducción de la pobreza y la paz mundial, la reflexión sobre el cambio de paradigma del propio concepto de retribución es clave para promover el crecimiento económico, el empleo pleno y productivo y, el trabajo decente para todos, como determina Naciones Unidas en la formulación de su objetivo de desarrollo sostenible número 8 . Y, esto es importante, porque, para combatir la pobreza, se hace necesario crear empleos estables y bien remunerados, amén de mejorar las condiciones laborales de unos 780 millones de mujeres y hombres que trabajan, pero no ganan lo suficiente para que ellos y sus familias puedan salir de la pobreza (Naciones Unidas, 2019).

En comparación con otras áreas y políticas de recursos humanos, y a pesar de la relevancia de estos asuntos, son escasas las investigaciones que dedican atención al poder que puede llegar a tener una adecuada concepción de la política retributiva como elemento indispensable en el crecimiento y desarrollo económico de las empresas y, por ende, de las naciones. El número de artículos dedicados a la selección, valoraciones del desempeño y rotación de empleados es muy superior a los estudios específicos de retribución y, muchos de ellos, centrados exclusivamente en la retribución variable de dos colectivos críticos: directivos y vendedores (Gómez Mejía et al., 2016).

En este contexto, se ha prestado poca atención a la evolución del concepto de retribución en clave a dar respuesta a las expectativas que el empleado del siglo XXI concibe como un "trabajo decente". En el presente artículo se analizará la posibilidad y conveniencia de incluir dichas expectativas en la propia ampliación del concepto. En primer lugar, se enunciará el objetivo del trabajo, y se argumentará, asimismo, los motivos por los cuales se considera que las expectativas deberían formar parte de una definición de retribución más amplia, sostenible en el tiempo e inclusiva. Posteriormente, se presentarán algunas decisiones laborales que ponen de manifiesto la importancia de tener en cuenta las expectativas de la población trabajadora en la realidad empresarial. Finalmente, se expondrán las conclusiones proponiéndose también, nuevas líneas de investigación. 


\section{SOBRE EL PROPIO CONCEPTO DE RETRIBUCIÓN}

Si se hace una retrospección a la narrativa sobre la época romana, por encuadrar el análisis terminológico desde una perspectiva histórica, la palabra salario tiene su origen en la sal que usaban los romanos, como forma de pago a los legionarios (Pérez Sánchez, 2018). Según el Diccionario de la Real Academia Española, la entrada sobre el término salario significa "cantidad de dinero con que se retribuye a los trabajadores por cuenta ajena". Siguiendo la definición más estricta del término, se le podría identificar con el salario fijo monetario que se percibe mensualmente. Sin embargo, esta definición parece alejada de poder explicar la realidad del mercado laboral.

Los términos retribución o remuneración se usan actualmente con un significado más amplio que salario, incluyendo no solo la retribución fija en metálico, sino también la retribución variable, otras retribuciones fijas o variables en instrumentos financieros como acciones u opciones sobre acciones, y, beneficios que no son estrictamente monetarios: coche de compañía, seguro médico, seguro de vida, etc. Dependiendo del sector, empresa y nivel profesional, la importancia de las retribuciones en especie o beneficios puede ser muy relevante. En este sentido, se puede definir la remuneración como los pagos directos o indirectos a empleados de bonus, acciones o beneficios (Gerhart \& Milkovich, 1992). Esta definición incluye beneficios cuantificables e individualizables, que en muchos países son objeto de tributación personal.

A estas definiciones más amplias, los manuales académicos sobre gestión de recursos humanos (Milkovich \& Newman, 2004; Gómez-Mejía et al., 2016; Armstrong et al., 2017) se refieren con el término de "retribución y/o compensación total", añadiendo beneficios más difícilmente cuantificables como son las instalaciones de diverso tipo (cafetería, comedor, gimnasio, etc.), los servicios a disposición de los trabajadores (lavandería, guardería), etc. El beneficio derivado de estos elementos que conforman los paquetes de compensación flexibles, puede no ser monetario. Por ejemplo, una guardería de empresa, aunque los gastos corran a cuenta de los empleados, puede facilitar el equilibrio entre vida laboral y personal por la comodidad que supone tener este servicio accesible durante el horario de oficina y cómodamente situada cerca del lugar de trabajo. Esta ventaja de conveniencia es difícilmente cuantificable y puede no estar sometida a tributación por parte de los empleados.

Desde el marco de una fuerza laboral globalizada, la diversa fiscalidad y regulación legal al respecto de cada país, resulta determinante para la aplicación de la política retributiva en cada caso. Así, es normal que las empresas multinacionales definan normas corporativas que se adaptan en cada país en las que tienen presencia de acuerdo con las leyes y prácticas de cada lugar. Hay países que tienen una 
normativa fiscal que favorece los vehículos de empresa, los planes de ahorro para la jubilación u otras retribuciones en especie. Es lógico que las empresas adopten localmente las políticas de remuneración apropiadas para optimizar la inversión y la satisfacción obtenida por los empleados.

En este sentido, también es una práctica cada vez más extendida en el mercado la implantación de planes de retribución flexible que permiten a los trabajadores elegir los beneficios de acuerdo con sus preferencias y aprovechar la capacidad de compra a gran escala de la empresa.

Los esfuerzos de las empresas para encontrar la forma óptima de retribución derivan de la creciente importancia de las dificultades que encuentran en el mercado actual para atraer al talento que necesitan. Numerosos estudios recientes indican que los CEOs a nivel mundial consideran el talento como uno de sus principales retos para lograr el éxito de su organización, por ejemplo, el estudio del Conference Board (Charam et al., 2015). Como señalan Milkovich y Newman, (2004): "es la competitividad por el talento la que está exigiendo a las empresas definir su estrategia de retribución en sentido amplio para resultar atractivas a los candidatos deseados". Esta estrategia debe ajustarse a cada empresa y modelo de negocio

Uno de los sectores con mayor escasez de talento es el de alta tecnología. Las empresas tecnológicas punteras como Google, Netflix o Apple, están revolucionado los beneficios para empleados en la guerra por retener el talento. Estas empresas, están ofreciendo todo tipo de ventajas, desde comida gratuita hasta días de descanso ilimitados. La creatividad para distinguirse de la competencia ha provocado iniciativas novedosas: entorno saludable, promover las oportunidades de crecimiento profesional o la comunicación bidireccional con los trabajadores.

Las iniciativas para crear un entorno saludable incluyen clases de yoga, gimnasio, servicio de fisioterapia, mobiliario adaptado (mesas ajustables que permiten trabajar de pie o sillas ergonómicas) y, en general, aspectos que mejoren la salud de los empleados en el lugar de trabajo. Por ejemplo, en Facebook se prestan bicicletas de forma gratuita para que los empleados puedan dar paseos en los alrededores de la oficina (Adams, 2014). El entorno saludable presenta un retorno sobre la inversión inmediato en forma de disminución del absentismo laboral por enfermedad y mayor rendimiento.

Uno de los beneficios más apreciados por los trabajadores es un entorno que promueva las oportunidades de aprendizaje y crecimiento profesional. Este entorno se puede facilitar mediante la definición de itinerarios profesionales claros, plataformas web de autoformación o, programas de mentores. Otro de los aspectos que valoran las nuevas generaciones es el respeto/reconocimiento y, el sentirse escuchados. En este ámbito, las empresas organizan reuniones con empleados, encuestas y planes de comunicación que destacan la influencia de los colaboradores sobre la marcha de la compañía (Smith, 2016). 
En este contexto de retribución total, Armstrong et al., (2017) destaca la importancia de considerar todos los aspectos retributivos como un todo coherente integrado en el resto de las iniciativas de recursos humanos para alcanzar la motivación, compromiso y desarrollo de los empleados. Milkovich y Newman (2004) ya propusieron un concepto que denominaron retornos totales (total returns) que tiene en cuenta otros beneficios asociados a la relación laboral: la seguridad en el empleo, el reconocimiento y estatus asociados a un puesto de trabajo, las oportunidades de aprendizaje o, el reto que supone el contenido del trabajo que se desempeña.

WorldatWork (2018), la reputada asociación de profesionales de recursos humanos considera que la retribución total está compuesta por seis componentes: retribución, beneficios, efectividad en el equilibrio profesional y personal, reconocimiento, gestión del rendimiento y desarrollo del talento; que, en su conjunto, definen la estrategia de una organización para atraer, motivar, fidelizar y comprometer a los empleados.

En resumen, la definición de salario se aleja cada vez más del significado puramente monetario. Por ejemplo, en China, el término tradicional de retribución está siendo desplazado por el término dai yu que se refiere a cómo la empresa trata o cuida a los trabajadores, en un nuevo indicio de que el término se entiende cada vez de una manera más amplia e integral (Milkovich \& Newman, 2004).

Actualmente, las empresas definen el concepto de retribución de una forma más o menos amplia dependiendo de su estrategia de atracción y fidelización de talento. Y esto, coincide de pleno con lo que Naciones Unidas (2019) en la formulación de su objetivo de desarrollo sostenible número 8 , entiende por crear "trabajo decente": puestos por los que se perciba un ingreso justo; seguridad en el lugar de trabajo; protección social para las familias; un trabajo que ofrezca mejores perspectivas de desarrollo personal y profesional, y que, favorezca asimismo la integración social.

El planteamiento de este trabajo, aboga para que, estas expectativas, sean consideradas en la definición de retribución porque, además de ayudar a explicar mejor el comportamiento de las empresas y trabajadores en la realidad laboral, se contribuiría con ello, como así lo espera también la población trabajadora del siglo XXI, a promover el crecimiento económico sostenido, inclusivo y sostenible, el empleo pleno y productivo y, el trabajo decente para todos.

A continuación, se presentan algunas teorías y situaciones en las que las expectativas de los trabajadores sobre su situación laboral desempeñan un papel relevante en la toma de decisiones empresariales. 


\section{DISCUSIÓN SOBRE LA IMPORTANCIA DE LAS EXPECTATIVAS EN EL ÁMBITO RETRIBUTIVO}

La importancia de prever el futuro fue documentada por Aristóteles (Versión de Massanés, 1954) cuando describió cómo Tales de Mileto obtuvo un merecido crédito por predecir un año de abundante recolección de aceite. Recientemente, la importancia de las expectativas en las decisiones retributivas fue apuntada por Milkovich y Newman (2004) quienes destacaban la necesidad de calcular el valor presente de las retribuciones esperadas en el momento de tomar decisiones sobre la carrera profesional. En su clásico libro, "Compensation", explican cómo una prestigiosa consultora (Andersen Consulting) argumentaba que el relativamente bajo salario de entrada se compensaba con incrementos de salario superiores en el futuro (para los trabajadores con un buen rendimiento se entiende). En realidad, la consultora estaba vendiendo el valor actual de las posibles retribuciones futuras en un inteligente ejercicio que demuestra la importancia de gestionar las expectativas.

La importancia de las expectativas para explicar el comportamiento de las personas en el mercado laboral ha sido objeto de diversos estudios (Donovan, 2001; Yu, \& Guo, 2012; Renko, Kroeck, \& Bullough, 2012; Purvis, Zagenczyk, \& Mccray, 2015). Desde una perspectiva macroeconómica, las expectativas cobraron relevancia en el debate sobre la efectividad de las políticas públicas para lograr el pleno empleo. Las discusiones se centraban en la capacidad de las políticas económicas expansivas para crear una ilusión monetaria que redujese el nivel de desempleo. La introducción del concepto de expectativas racionales (Muth, 1961) demostró que este efecto no era sostenible en el largo plazo ya que los individuos aprendían y formaban sus expectativas de una forma racional.

Posteriormente, desde una perspectiva microeconómica, Mortensen (1970) propuso una teoría que ponía de relieve cómo los profesionales se enfrentan a un proceso de búsqueda de trabajo en situaciones de incertidumbre. Según este modelo, cada profesional evalúa el mercado y su propia situación, y reflexiona sobre si un mayor tiempo de búsqueda de trabajo, se puede ver recompensado con una posición mejor remunerada. Un profesional que reciba una oferta de trabajo con un salario determinado evaluará, antes de aceptar, si puede encontrar un salario mayor en caso de continuar la búsqueda.

En la actualidad, Anta y Stein (2017) proponen una nueva concepción sobre la retribución, entendiendo por ésta: "el conjunto de los rendimientos recibidos por el profesional por el trabajo realizado y los objetivos cumplidos, así como el valor percibido de la relación esperada con su empleador". Esta definición incluye los beneficios financieros y no financieros, los beneficios sociales, las oportunidades de desarrollo y el contenido del puesto de trabajo, el ambiente de trabajo, las expectativas de carrera, etc. Además, considera las expectativas salariales en 
forma de "valor percibido de la relación esperada con su empleador", es decir, incluye los ingresos futuros esperados matizados por el nivel de riesgo que cualquier estimación conlleva.

Una definición del concepto de retribución que tenga en cuenta el futuro ayuda a explicar muchas de las situaciones que vivimos en el mercado laboral. A continuación, se analizan algunos aspectos que ponen de manifiesto la importancia de las expectativas en el comportamiento de los trabajadores. En primer lugar, se exponen los dos modelos empíricos que presentan mayor poder explicativo al efecto, como son la teoría psicológica de expectativas para motivar y retribuir a los empleados (Vroom, 1964) y, el impacto que la atención a las expectativas de los trabajadores puede tener en los resultados empresariales, encuentran su mejor caja de resonancia en el modelo explicativo sobre el "contrato psicológico" (Cegarra et al., 2013). Seguidamente, y a la luz de dichas teorías, se analizarán situaciones prácticas de la vida laboral como, por ejemplo, la contratación, la expatriación, la decisión de cursar un MBA, etc.

\section{MARCO TEÓRICO}

\section{LA TEORÍA DE LAS EXPECTATIVAS LABORALES}

La primera teoría sobre las expectativas para motivar y retribuir a los empleados es la de Víctor H. Vroom, que establece que la fuerza de nuestra tendencia a actuar de cierta manera depende, de la magnitud de las expectativas que tenemos de obtener un resultado y de su atractivo. Los individuos se sentirán más motivados a esforzarse, cuando crean en estas tres relaciones claves (Vroom 1964).

- EXPECTATIVA: probabilidad percibida de que el esfuerzo conduce a una actuación de éxito y/o una buena evaluación de su desempeño.

- INSTRUMENTALIDAD: creencia de que una buena evaluación les proporcionará las recompensas esperadas.

- VALENCIA: que las recompensas organizacionales otorgadas satisfacen sus necesidades o metas personales.

Porter y Lawler (1968), profundizan en las variables que influyen en el proceso de motivación (Miner, 2007) concluyendo que, aumentará la motivación del trabajador si se fortalecen los vínculos correlativos entre ellas y, siempre y cuando, los individuos perciban con claridad la relación entre el esfuerzo y el desempeño, al igual, que la evaluación con la recompensa. Y si ésta, finalmente, es la que los empleados, aspiraban obtener en cantidad, calidad y cualidad. 
Como se puede deducir de esta teoría, la retribución debe adquirir un enfoque amplio, total y flexible, que contemple todas estas expectativas en las que se vinculan a la compensación laboral, el reconocimiento personal y profesional, la evaluación objetiva del desempeño, incluyendo también, la valoración del esfuerzo realizado por el empleado, no únicamente los objetivos cumplidos.

\section{EL CONTRATO PSICOLÓGICO}

Otra de las teorías que tiene en cuenta la importancia de las expectativas en las relaciones laborales es la teoría del "contrato psicológico" (Rousseau, 1993). Este acuerdo tácito, se puede definir como el conjunto de compromisos que espera el trabajador de la empresa y viceversa. Este contrato se comienza a forjar desde las relaciones previas a la contratación entre los candidatos y la empresa, y evoluciona a lo largo de toda la relación laboral.

Si se analiza en mayor profundidad los antecedentes de una contratación, ya durante el proceso de selección los profesionales tienen contactos con la organización a través de distinto medios (anuncios, página web corporativa, etc.) y, posteriormente con responsables de recursos humanos, directivos o profesionales de la empresa para la que van a trabajar. En este proceso de conocimiento mutuo, previo a la contratación, cada candidato se forja unas expectativas sobre el funcionamiento de la compañía y el futuro que le espera. En estos encuentros, las expectativas sobre la compensación del trabajo se pueden formar de distintas maneras (Rousseau, 1993):

- Formal: por escrito en la carta de oferta o en el contrato de trabajo. En esta categoría podemos encontrar incrementos salariales pactados o pagos garantizados para períodos futuros.

- Explícita: promesas realizadas por algún responsable de la empresa de forma verbal que no se incorporan al contrato ni en la carta de oferta.

- Implícita: compromisos que no se adquieren de una manera explícita, sino que resultan del contexto de una conversación.

- Circunstancial: Aquellas que se crea el trabajador por lo que "dicen otros compañeros", "han oído" o "todo el mundo sabe", etc.

El contrato psicológico se basa en las promesas que cada uno percibe como exigibles. La ruptura de una promesa se puede producir independientemente de su origen, ya sea formal, explícito, implícito o circunstancial. Así, puede que una carta de oferta no esté bien redactada y provoque diferencias de interpretación a la hora de aplicarlo. Por otra parte, las promesas explícitas están expuestas al malentendido por el hecho de no estar documentadas. Por este motivo, los departamentos de recursos humanos suelen recomendar formalizar los compromisos. En último lugar, 
las interpretaciones sobre promesas contraídas de forma implícita o, circunstancial, son frecuentemente objeto de discusiones posteriores por su falta de concreción. En este sentido, se debe ser muy cauto a la hora de realizar cualquier comunicación con los trabajadores (comunicar la valoración del desempeño, la revisión salarial o, en el momento de hacer una oferta) para ser claro en la fijación de expectativas.

Muchas rupturas del contrato psicológico tienen su origen en asunciones no explícitas. Los directivos, deben entender que una de sus funciones clave es gestionar las expectativas, $y$, en concreto, clarificar lo que piensan que sus equipos deben conseguir, las competencias que deberían poseer y, los valores que deberían defender (Armstrong et al., 2017).

Los candidatos que aspiran a ser contratados por una empresa saben que su conocimiento de la organización es limitado y que deben interpretar con cautela la información obtenida pues, en ningún caso, se puede incluir toda esta información en un contrato laboral. Es inevitable que cada persona se forje expectativas sobre los detalles de la posición que va a desempeñar, la cultura de la empresa, y por supuesto, sobre las posibilidades de aumento de retribución en los próximos años. En este sentido, en la entrevista final antes de la adscripción al puesto de trabajo, es aconsejable hacer una recapitulación de los elementos esenciales del contrato psicológico para estar seguro de que no existen malentendidos. Una vez que se produzca la incorporación en la compañía, cualquier incumplimiento de las promesas será interpretada como una ruptura del acuerdo alcanzado (Tena, 2002).

En el ámbito de la compensación total, esta teoría del "contrato psicológico", refuerza la importancia de las expectativas a lo largo de la relación laboral y, por tanto, ayuda a entender el comportamiento de los trabajadores en situaciones como las que se describen a continuación.

\section{ANÁLISIS DE SITUACIONES LABORALES, A LA LUZ DE LA TEORÍA DE LAS EXPECTATIVAS}

\subsection{El momento de la contratación}

El momento de la contratación es uno de los más críticos en el establecimiento de las expectativas de los trabajadores. El contrato laboral supone la formalización de la contratación. En ocasiones, puede existir una carta de oferta, previa a la firma del contrato, que resuma las condiciones que se reflejarán posteriormente en el mismo. El tipo de contrato varía según las empresas, sector, nivel profesional, convenio aplicable, etc. El contrato puede ser sencillo (cumpliendo los mínimos legales) o, tener una mayor extensión reflejando todas las características particulares diferentes de las legalmente aplicables o impuestas por convenio. En el caso 
de los directivos, los contratos suelen ser extensos y pueden incluir cláusulas como blindajes, no competencia post contractual, etc.

Como se señaló anteriormente, los contratos son en todo caso, incapaces de formalizar y/o explicitar absolutamente, todos los elementos que forjan el contrato psicológico con un trabajador pudiendo darse posteriormente serios equívocos o falsas interpretaciones. A continuación, se ilustra con un ejemplo cómo se producen los malentendidos.

Imaginamos la situación de un vendedor, que firma un contrato con una empresa que tiene su sede en la ciudad de Santander, con una cláusula de disponibilidad para viajar dentro de España de acuerdo con las necesidades del puesto de trabajo. Es muy difícil estimar e incluir en el contrato el número de días o los destinos de estos viajes, ya que, dependerá de los clientes con los que se establezca relación comercial. Puede darse la circunstancia de que el trabajador gane un gran contrato con un cliente que esté establecido en la misma ciudad en la que reside, Santander, y que circunstancialmente no tenga que viajar ese año. Por el contrario, puede que el contrato comercial, lo gane con una empresa que tiene su sede en Cádiz y, tenga que desplazarse con mayor frecuencia de lo previsto. La clave no está en el contrato (expectativas formalizadas), sino en la conversación mantenida durante la firma.

Supongamos, además, que el trabajador hubiese preguntado durante la última entrevista si se podía estimar el número de días de viaje por año. Una respuesta clara hubiese sido: "según pone en el contrato, deberás viajar de acuerdo con las necesidades de tu trabajo, no puedo concretar un número de días". Una respuesta un poco más arriesgada hubiese sido: "normalmente tus compañeros están viajando unos 20 o 30 días al año". En esta respuesta ya se está produciendo un compromiso implícito que puede dar problemas futuros. Otra posible respuesta sería: "no te preocupes, que si logras un gran contrato fuera de Santander, repartiremos los viajes entre los miembros del equipo para asegurar que no viajas más de 50 días al año". En este caso, ya hay una promesa explícita no documentada que formará parte, sin duda, del contrato psicológico con el trabajador.

Como se puede apreciar en el ejemplo anterior, los contratos laborales están potencialmente sujetos a expectativas que no pueden quedar formalmente documentadas. Lo mismo ocurre con las revisiones futuras de salario fijo, son otro buen ejemplo esto. Las subidas salariales dependerán de diversos factores que son imposibles de estimar en el momento de la contratación: rendimiento del trabajador, marcha de la empresa y presupuestos salariales aprobados cada año, etc.

En el ámbito retributivo, el área de discusión más frecuente es la retribución variable. A continuación, se analiza en mayor detalle estas situaciones de negociación sobre las expectativas de percepción de incentivos. 


\subsection{La retribución variable}

Éste, es uno de los ámbitos más frecuentemente sujetos a una ruptura del contrato psicológico. Como su propio nombre indica, este tipo de retribución depende, entre otras variables, de los resultados del trabajador. Sin embargo, el pago está condicionado de forma crítica por la política de fijación de objetivos.

Muchas empresas definen una retribución variable teórica o target como la retribución correspondiente a un cumplimiento del 100\% de los objetivos. Si el cumplimiento de los objetivos es mayor o menor, la retribución variable real a pagar, será igualmente mayor o menor. Un análisis histórico de los últimos periodos puede demostrar el porcentaje de personas que ha alcanzado el target. Existen diferentes casos, entre los que se pueden destacar dos escenarios diferentes:

- Escenario de objetivos realistas: se alcanzan los objetivos normalmente (es decir, aproximadamente en el 50\% de los casos). Los objetivos se definen de forma alcanzable y realista, y el bono target es conseguible en condiciones normales de mercado.

- Escenario de objetivos ambiciosos: en estos entornos, en media, solo el 15-25\% de los empleados, logran el 100\% de los objetivos, es decir, los objetivos se definen de forma aspiracional y la remuneración target está destinada a un colectivo minoritario y con excepcional rendimiento.

En el momento de realizar una oferta de trabajo, la retribución variable se suele acordar en términos target. Es lógico que los profesionales pregunten por la política de definición de objetivos para entender, con qué probabilidad pueden razonablemente esperar recibir la retribución pactada. Durante la contratación, se producirán conversaciones alrededor de la cultura de fijación de objetivos que formarán parte de las expectativas no formalizadas del contrato psicológico.

Además, la retribución variable puede asignarse discrecional, total o parcialmente. En estos sistemas, la decisión sobre la cantidad de retribución variable a pagar depende de los directivos. Los departamentos de recursos humanos suelen recomendar que se realice una argumentación objetiva del pago propuesto para cada individuo teniendo en cuenta el trabajo de cada uno y, la aportación al éxito de la organización (Miner, 2007).

En la práctica, una óptima distribución de los bonos puede quedar sustituida por prácticas recurrentes que conforman la cultura de la empresa. En algunos casos, la retribución variable discrecional puede convertirse en un fijo no consolidado. En otras ocasiones, la retribución variable se fija con un mismo criterio para todos los miembros de un equipo; por ejemplo, mantener, incrementar o disminuir en igual proporción respecto al variable del año anterior. 
En conclusión, cualquier sistema de retribución variable, sea discrecional o no, está sujeto a las expectativas que la aplicación en la práctica se crea en la mente de los trabajadores, sin embargo, es un elemento clave y crítico, en la conformación del contrato psicológico entre el trabajador y la empresa.

\subsection{Cursar un MBA}

Una vez concluidos los estudios universitarios, y normalmente, tras algunos años de experiencia en el mercado laboral, cursar un MBA (Master in Business Administration) se presenta como una oportunidad de formarse para acceder a puestos gerenciales que exigen la comprensión global de las distintas funciones de una empresa. Las escuelas de negocio recomiendan tener algunos años de experiencia antes de acceder al programa. Sin embargo, hay estudios que analizan la relación entre los salarios obtenidos al finalizar el MBA con la experiencia previa y que vienen a sugerir, que no existe una correlación relevante entre estos dos conceptos (Yeaple et al., 2010).

El MBA ofrece la posibilidad de cambiar o impulsar la carrera profesional reforzando las habilidades de gestión. Típicamente, es un trampolín para acceder a puestos de dirección por todas las ventajas que estos programas ofrecen: conocimientos en materias de gestión, trabajo en equipo, adquisición de competencias a través del aprendizaje práctico (método del caso), etc. Adicionalmente, el MBA ofrece la oportunidad de conectar con personas en la misma situación, diferenciarse respecto a otros candidatos, fomentar la creatividad y, el conocimiento del mercado, entre otros aspectos.

Las escuelas de negocio que tienen programas de MBA compiten por atraer a los mejores candidatos con el objetivo de elevar el nivel de sus estudiantes y mejorar así, las estadísticas que anualmente se calculan para hacer los rankings retributivos anuales (Schenk, 2017). En un informe realizado por U.S. News en el que participaban 114 escuelas de negocio de Estados Unidos, la retribución total (incluyendo salario fijo y variable) pagada en 2017 a los estudiantes recién egresados, ascendía a 105.146\$ (Kowarski, 2018). La escuela con mayor salario fue la de Standford (California), con cerca de 174.000\$ anuales, seguida de Harvard, Wharton y Columbia con salarios superiores a los $160.000 \$$ anuales.

El estudio revela una diferencia significativa respecto al salario medio para los recién egresados de los MBA que menos retribución obtienen, y cuya media, se sitúa en aproximadamente 58.000\$ anuales. Los salarios dependen también del sector en el que se trabaje. El estudio apunta que los sectores que ofrecen los salarios más altos en la actualidad son los de consultoría, servicios financieros y tecnología.

Un MBA es considerado una inversión en la carrera profesional. Como en cualquier inversión, se puede calcular el Retorno sobre la Inversión (ROI) de 
diferentes formas. La forma más habitual de calcular el ROI es comparar el salario post-MBA con el salario pre-MBA calculando asimismo, el número de años que se tarda en recuperar el coste de la inversión o el valor presente de la decisión en 10 o 20 años (Iqbal, 2015).

La ciencia de cuantificar el retorno sobre la inversión de un MBA es una ciencia inexacta, pero según la Alumni Perspectives Survey que, contó con la participación de 4.000 estudiantes en 2012, en media, habían recuperado su inversión en 4 años (Lightfoot, 2013).

En 2014, se realizó otro estudio en 46 escuelas de negocio en Europa, cuyos resultados indican que estudiantes de 12 instituciones fueron capaces de duplicar su retribución tras su paso por el MBA. La media de incremento salarial se sitúo en un $85 \%$. Los aumentos también variaban según el país y el sector de actividad. Los mayores aumentos se detectaron en las siguientes escuelas que se muestran como ejemplo de los incrementos salariales conseguidos (Dhoul, 2015): Vlerick Business School (de 36.500€ a 93.000€), St. Gallen MBA (de $52.000 €$ a $130.000 €$ ) y University of Edingburgh Business School (de $40.000 €$ a $98.500 €)$.

Según esta misma encuesta, los mejores incrementos salariales de las mejores escuelas europeas en 2014 fueron: London Business School (de 57.300€ a $118.650 €$ ), INSEAD (de 71.000€ a $120.563 €$ ) y SAID Business School (de $58.000 €$ a $99.734 €)$.

Un ejemplo de cómo se argumenta el cálculo del retorno sobre la inversión se puede encontrar en el informe de tendencia de salarios de TopMBA.com, que lo explica de la siguiente forma: "En media, los aplicantes al MBA en la región están ganando 40.000\$. Si tomamos los gastos de matriculación de un programa de dos años en los Estados Unidos podrían estar en torno a los 100.000\$, y teniendo en cuenta el coste de oportunidad de dejar de trabajar durante dos años (...). Ahora, imaginando que eres una persona de 28 años y que vas a trabajar 25 años más, con un salario creciendo en media un $7 \%$ por año (...) El valor presente de la inversión estará probablemente entre los 2 y los 3 millones de dólares durante tu vida profesional" (Iqbal, 2013).

Otro estudio realizado entre 550 estudiantes de MBA comparó el salario pre-MBA con el salario recibido cinco años después de terminar los estudios, concluyendo que los salarios habían crecido un $80 \%$. Estos datos son consistentes con estudios anteriores publicados por el mismo autor (Yeaple, 1994; 2014).

En conclusión, con todos estos datos publicados, resulta evidente que las expectativas de salario, también están en la mente de los egresados y/o, profesionales, a la hora de evaluar la decisión de realizar un MBA. Es lógico pensar que, ellos, tienen en cuenta todos estos datos salariales publicados por los distintos estudios, a la hora de negociar sus paquetes retributivos en las empresas. 


\subsection{Cambios de trabajo asociados a una reducción de salario}

Otro momento importante en la carrera profesional es el cambio de trabajo. La creencia de que solo cambiamos de trabajo si nos ofrecen un salario superior es antigua y, no se corresponde con la realidad del mercado laboral actual. Actualmente, existen diferentes motivos por los que una persona está dispuesta a cambiar de trabajo por una retribución inferior.

Las motivaciones se encuentran, desde buscar un mayor equilibrio entre la vida profesional y personal, hasta hacer un trabajo que te autorrealice. Desde luego, levantarte todas las mañanas e ir a un trabajo que te gusta, te ayuda a recordar que los sacrificios en el corto plazo pueden verse recompensados en el largo plazo (Clayton, 2018). El punto hasta el que nos sentimos anclados a nuestro salario depende enteramente de nosotros (Walker, 2018).

Se dice que un profesional se encuentra en una "jaula de oro" cuando no es feliz en su puesto de trabajo, pero no está dispuesto a que se reduzca su retribución de ninguna manera. Este fenómeno es común en industrias en declive, cuyas remuneraciones fueron elevadas en el momento más alto del ciclo del producto, servicio o sector, pero que ahora se ven superadas por nuevas tecnologías o, modelos de negocio.

La incorporación de expectativas retributivas en un sentido amplio resulta fundamental en estos casos para tomar la decisión de reducir el salario para entrar en una industria con mayor proyección e incrementos futuros. Cuando solo se tiene en cuenta el salario fijo inicial, la visión sobre las consecuencias de cada alternativa resulta incompleta. En la realidad laboral se producen muchos cambios de trabajo asumiendo una reducción salarial monetaria inicial, para lograr otro tipo de beneficios, sugiriendo que nuestra propuesta explicitada al inicio del trabajo es acertada.

\subsection{La expatriación}

Los traslados internacionales son cada vez más frecuentes en el contexto de una economía globalizada, en la que es frecuente que surja una necesidad de negocio que requiera el desplazamiento de un profesional durante un período de tiempo determinado. En la medida en que esta vacante no pueda ser ocupada por un trabajador local, la empresa estará dispuesta a incentivar a los empleados que estén disponibles para mudar temporalmente su lugar de residencia. Esta situación es frecuente cuando una empresa se expande a nuevos mercados y necesita del conocimiento de sus trabajadores en el extranjero o necesita personas de confianza en puestos clave (Gómez Mejía et al., 2016). 
Una expatriación, se puede definir como un traslado internacional con compromiso de retorno al país de origen, que se incentiva con ciertas compensaciones y ayudas temporales durante el tiempo que dura el desplazamiento.

Por tanto, la compañía subvenciona una serie de conceptos al empleado para incentivar el traslado familiar y lograr los siguientes objetivos:

- Mantener el nivel adquisitivo del país de origen.

- Cubrir los costes adicionales en los que incurrirá el empleado por causa de su desplazamiento.

- Compensar del esfuerzo que supone el cumplimiento de objetivos durante el desplazamiento.

En este escenario, el salario de partida en el país de origen se ve aumentado durante el período que dura la expatriación con complementos, beneficios y ayudas para el expatriado y su familia, entre los que se puede destacar los siguientes: complemento salarial por diferencial de coste o calidad de vida entre el país de origen y destino, ayuda para pagar la vivienda y el colegio de los hijos, cobertura de seguro médico y seguro de vida, pago de los costes de la mudanza y gastos de instalación, ayuda para cubrir la asesoría fiscal y migratoria, formación o búsqueda de empleo del cónyuge, asunción de los mayores impuestos a pagar por el expatriado derivado de su desplazamiento, etc.

En consecuencia, el coste de una expatriación supone entre 1,5 y 4 veces el gasto de un empleado local según la consultora de recursos humanos Mercer en su informe Estudio mundial sobre asignaciones internacionales 2008, elaborado con más de 100 multinacionales (Espinosa, 2009).

Normalmente, las empresas formalizan en la misma carta de desplazamiento las condiciones retributivas de retorno para fijar las expectativas salariales de antemano. Sin embargo, las expectativas que se forjan de manera implícita y circunstancial, pueden llegar a ser demasiado elevadas.

Es frecuente, que se provoque una situación que comúnmente se denomina el "síndrome del expatriado". En estos casos, el expatriado, aun habiendo firmado que su salario de retorno volvería a estar en el entorno del salario de referencia previo a su desplazamiento, puede no aceptar de buena gana la reducción de su paquete retributivo en el momento de retorno. Un fenómeno similar puede afectar a su percepción del puesto de trabajo de retorno asignado a su vuelta y a sus aspiraciones profesionales.

En conclusión, el síndrome del expatriado es otro ejemplo sobre la importancia de la gestión de las expectativas en materia retributiva. 


\section{CONCLUSIÓN}

La actual guerra por el talento está agudizando el ingenio de las organizaciones para resultar atractivas y tener una propuesta de valor diferenciada respecto a las empresas por las que compiten por el talento.

La retribución total, es un concepto sobre el que actualmente no existe unanimidad. Las empresas definen el término de acuerdo con su estrategia de atracción y fidelización de talento. Sin embargo, se puede concluir que tanto las empresas como los trabajadores están teniendo muy en cuenta las expectativas a la hora de tomar decisiones en el ámbito laboral.

Así, la decisión de cursar un MBA es el caso que más estudios cuantitativos y literatura acumula poniendo de manifiesto que los profesionales, tienen en cuanta un horizonte temporal amplio en el momento de tomar esta decisión. Los estudiantes consideran el salario esperado tras terminar el máster y, calculan el retorno sobre la inversión que supone.

Pensemos en un expatriado que está negociando sus condiciones de desplazamiento, ¿es razonable pensar que tratará de negociar también sus condiciones de retorno? En el momento de acordar las condiciones de incorporación a una empresa, ¿piensan los trabajadores en cómo evolucionarán sus condiciones en el futuro? Parece difícil imaginar que en estas negociaciones no se tenga en cuenta el futuro y por tanto sus expectativas.

Estos casos presentados, demuestran que la definición de retribución puede llevar a decisiones racionales diferentes según incorpore o no las expectativas de los trabajadores. Por tanto, su inclusión en la definición de retribución resulta, además de pertinente, muy relevante.

En conclusión, las decisiones que se toman en la realidad laboral parecen explicarse mejor con una definición amplia del concepto de retribución total que incorpore dichas expectativas y que garanticen la creación de "trabajo en condiciones decentes" como lo requiere la ONU (2019) en la declaración de su objetivo de desarrollo sostenible número 8: "Promover el crecimiento económico sostenido, inclusivo y sostenible, el empleo pleno y productivo y el trabajo decente para todos".

\section{FUTURAS LÍNEAS DE INVESTIGACIÓN Y LIMITACIONES DE ESTE ESTUDIO}

Se podría profundizar en esta línea de investigación para llegar a una definición de consenso que sea cercana a la práctica empresarial actual. Además, este ámbito presenta líneas de investigación tanto desde la perspectiva del trabajador, como de las empresas en su estrategia de atracción y fidelización del talento. 
Primero, se podría identificar los distintos elementos que usan las empresas para atraer y fidelizar el talento porque, si bien todo lo expuesto sobre la teoría de las expectativas es relevante desde el punto de vista psicosocial, aunque el análisis de este artículo no sea jurídico, conviene resaltar la importancia de las repercusiones positivas que tiene la fiscalidad de los planes de compensación flexible que, sin duda, benefician tanto a empleados como a la propia organización, haciéndolos aún más atractivos.

Segundo, se podría estudiar empíricamente la cuantificación que distintos profesionales hacen de diversos elementos retributivos e identificar perfiles de profesionales según se sientan atraídos por un tipo de retribución u otra. De la misma forma, se podrían clasificar los sectores y empresas según el perfil de retribución que ofrecen. Esta vía de investigación podría corroborar la intuición de que las personas se sienten atraídas por sectores cuya oferta retributiva, entendida en términos amplios, se ajusta a su perfil.

Asimismo, una definición amplia sobre el concepto de retribución también abre el campo a la investigación de las técnicas de comunicación de la propuesta de valor al empleado, tanto internas como externas, para lograr atraer y fidelizar a los trabajadores que mejor encajen en cada organización.

\section{BIBLIOGRAFÍA}

Adams, S. (2014). America's Top Companies For Compensation And Benefits Forbes online version. Recuperado de: https://www.forbes.com/sites/susanadams/2014/05/23/americas-top-companies-for-compensation-and-benefits/amp/ (última consulta 14/10/19).

Anta, C. \& Stein, G. (2017). Cómo liderar personas con la retribución. políticas, criterios y valores. Madrid: Pearson Educación.

Aristóteles (1954). La Política. Versión de Natividad Massanés. Barcelona: Editorial Iberia (Obras maestras).

Armstrong, M. \& Taylor, S. (2017). Armstrong's handbook of human resource management practice. UK: Kogan page Publishers

Cegarra, D., Sánchez, M. E., Cegarra, J. G., ArizkurenR, A. \& Martínez, E. (2013). Cómo afecta la conciliación laboral y personal en la innovación de la pyme industrial. Estudio en el sector del metal de la región de Murcia. DYNA Management, 1, 1-11.

Charam, R. et al. (2015). People Before Strategy: A New Role for the CHRO. Harvard Business Review, July-August 2015 issue. Online version. Recuperado de: https://hbr.org/2015/07/people-before-strategy-a-new-role-for-the-chro (última consulta 8/12/19). 
Clayton, R. (2018). Can You Afford to Change Your Career? Harvard Business Review. Online version. Recuperado de: https://hbr.org/2018/08/can-you-affordto-change-your-career (última consulta 21/10/19)

Dhoul, T. (2015). MBA ROI: Top European Schools for an MBA Salary Boost Top MBA online version. Recuperado de: https://www.topmba.com/admissions/ financing-your-mba/mba-roi-top-european-schools-mba-salary-boost (última consulta 18/10/19).

Donovan, J. J. (2001). Work motivation. Handbook of industrial, work and organizational psychology, 2, 53-76.

Espinosa Romero, M. (2009). Los procesos de internacionalización empresarial y la gestión de personas. Madrid: EOI.

Gerhart, B., \& Milkovich, G. T. (1992). Employee compensation: Research and practice (CAHRS Working Paper \#92-26). Ithaca, NY: Cornell University, School of Industrial and Labor Relations, Center for Advanced Human Resource Studies. Recuperado de: http://digitalcommons.ilr.cornell.edu/cahrswp/311.

Gómez Mejía, L. Balkin, D. \& Cardy, R. (2016). Gestión de recursos humanos. 8o Ed. Madrid: Pearson Education S.A.

Gupta, N. \& Shaw, JD. (2014). Employee Compensation: The neglected area of HRM research. Human Resource Management Review, 24 (1), 1-4.

Iqbal, M. (2013). MBA Salary: Expectations vs. Reality, QS TOP MBA online version, Recuperado de: https://www.topmba.com/jobs/career-trends/mba-salary-expectations-vs-reality (última consulta 16/10/19).

Iqbal, M. (2015). How Do You Work Out the ROI of an MBA? Top MBA online version, Recuperado de: https://www.topmba.com/why-mba/how-do-you-workout-roi-mba (última consulta 23/10/19).

Kowarski, I. (2018). Find MBA Programs That Lead to Jobs, High Salaries. US News online version. Recuperado de https://www.usnews.com/education/ best-graduate-schools/top-business-schools/articles/2018-03-20/see-which-mbaprograms-lead-to-the-best-jobs-salaries (última consulta 25/10/19).

Lightfoot, L. (2013). Is studying an MBA a good investment? The Guardian online version, Recuperado de: https://amp.theguardian.com/education/2013/jan/22/ mba-postgrad-success (última consulta 28/10/19).

Milkovich, G. \& Newman, J. (2004). Compensation. 8th Edition, New Cork: McGraw-Hill

Miner, J. (2007). Organizational Behavior 4: From Theory to Practice. Armonk, NY: M.E. Sharpe.

Mortensen, D. (1970). Job Search, the Duration of Unemployment and the Phillips Curve. American Economic Review, 60, 847-862.

Muth, J. F. (1961). Rational Expectations and the Theory of Price Movements. The Econometric Society. Econometrica, 29 (3), 315-335. 
Naciones Unidas. Objetivos y metas de desarrollo sostenible. Recuperado de: https://www.un.org/sustainabledevelopment/es/ (última consulta 25/10/2019).

Pérez Sánchez, A.J. (2018, 21 de octubre). ¿Cuál era el sueldo y la alimentación de un legionario romano? [Entrada de Blog]. Blog "El reto histórico". Recuperado de https://elretohistorico.com/sueldo-alimentacion-legionario-romano/

Porter, L.W. \& Lawler, E. E. (1968). Managerial Attitudes and Performance. Homewood, Illinois: R. D. Irwin.

Purvis, R. L., Zagenczyk, T. J., \& McCray, G. E. (2015). What's in it for me? Using expectancy theory and climate to explain stakeholder participation, its direction and intensity. International Journal of Project Management, 33 (1), 3-14.

Renko, M., Kroeck, K. G., \& Bullough, A. (2012). Expectancy theory and nascent entrepreneurship. Small Business Economics, 39 (3), 667-684.

Rousseau, D.M. \& Parks, J. M. (1993). The contracts of individuals and organizations. Research in Organizational Behaviour, 15,1-1.

Schenk, K. (2017). Comparing MBA CoA to calculate ROI. Prodigy Finance online version. Recuperado de: https://prodigyfinance.com/resources/blog/comparingMBA-coa-ROI (última consulta 30/10/19).

Smith, L. (2016). Keeping Your Top Performers: Perks that Matter. Industry Week online version. Recuperado de: https://www.industryweek.com/compensation-strategies/keeping-your-top-performers-perks-matter (última consulta 24/10/19).

Tena, G. (2002). El contrato psicológico: relación laboral empresa-trabajador. Acciones e investigaciones sociales, 15, 85-107.

Vroom, V. H. (1964). Work and motivation. New York: John Wiley and Sons.

WALKER, R. (2018). You've Been Offered a Great New Job, but Is It Worth a Pay Cut? NY Times online version. Recuperado de: https://www.nytimes.com/2018/11/02/ business/new-job-pay-cut-worth-it.html (última consulta 11/10/19).

WorldatWork (2018). Total Rewards Concept and Model. WorldatWork web, https://www.worldatwork.org/total-rewards-model/

Yeaple, R.N. (2014). MBA Pay. Forbes online version. Recuperado de: https://www. forbes.com/sites/ronaldyeaple/2014/03/17/mba-alumni-profile-2/\#6315e43537c1 (última consulta 9/10/19).

Yeaple, R.N. (1994). The MBA Advantage: Why it Pays to Get an MBA. Massachusetts Adams Media Corporation.

Yeaple, R.N, Johnston, M.W \& Whittingham, K.L. (2009-2010). Measuring the Economic Value of Pre-MBA Work Experience. Journal of Education for Business, 85 (1), 13-20.

Yu, G. \& Guo, J. (2012). Research on Employee Motivation Mechanism in Modern Enterprises Based on Victor H. Vroom's Expectancy Theory. En Duysters, G., DeHoyos, A. \& Kaminishi, K. (eds). Proceedings of 9th International Conference on Innovation and Management (pp. 988-991). 